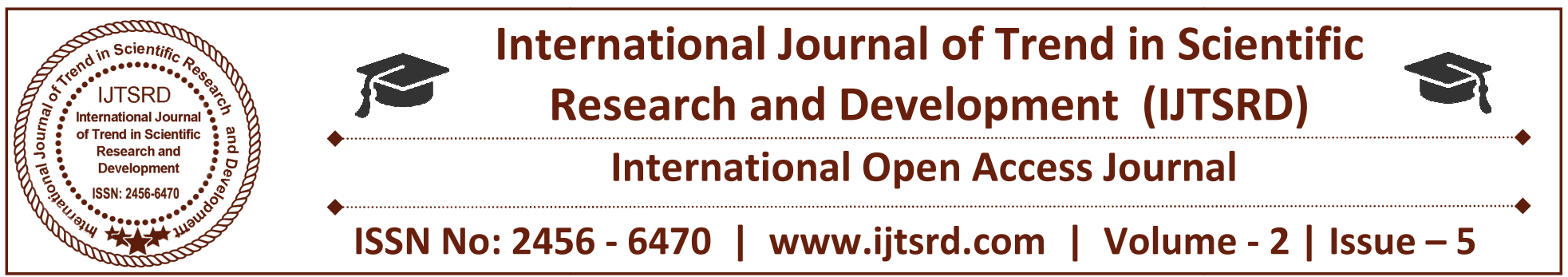

\title{
Real-Time Face Detection Security System using Haar Classifier Method
}

\author{
Ei Mon Phyo ${ }^{1}$, Nay Win Zaw ${ }^{2}$, Aye Aye Tun ${ }^{3}$ \\ ${ }^{1}$ Assistant Lecturer, ${ }^{2}$ Professor, ${ }^{3}$ Lecturer \\ Department of Electronic Engineering, West Yangon Technological University, Myanmar
}

\begin{abstract}
Face Detection is concerned with finding whether or not there are any faces in a given images. Security and surveillance are the two important aspects of human being. Face detection is very important because it is not being safe in human environment. So, Face Detection Security System is essential between individual in life. In the modern world everything is changed to provide a better life. So we were decided to develop the Real Time Face detection system. The importance of the face detection as it is essential for surveillance and real user interfaces security to the country. Face differ in skin colour, nose, eyebrows, chin between different people in humanity. In this paper we effort is to develop system about face detection security system in the real time.
\end{abstract}

KEYWORD: Haar classifier, Segmentation and Edge/Line Detection, Face Detection, Feature Extraction, Computer Vision

\section{INTRODUCTION}

In the modern world of living, new technologies are evolved every day. The most popular and widely use banking system, considering for smart higher security for superior life standard. Security System based on Face Detection and GSM for technology, which can be used in Banks, Security Offices and Homes for giving protection to expensive possessions. In this system, only the authorized person the valuable things like money, licenses and jewels from locker. Real time human identification systems are important for security, surveillance and biometric applications. [2]

\section{COMPUTER VISION}

Computer Vision is a library of programming functions mainly aimed at real-time computer vision.
In simple language it is library used for Image Processing. It is mainly used to do all the operation related to Images. The goal of Computer Vision is to emulate human vision using digital images through three main processing components, executed one after the other: Image acquisition, Image processing Image analysis and Understanding. Computer vision is closely linked with artificial intelligence, as the computer must interpret what it sees, and then perform appropriate analysis. [3]

\section{HAAR CLASSIFIER}

\subsection{What is Haar Classifier?}

Haar Cascade is basically a classifier which is used to detect the object for which it has been trained for, from the source. The Haar Cascade is by covering the positive image over a set of negative images. Haar cascade is an object detection algorithm. It is used to locate faces, pedestrians, objects and facial expressions in an image. Among them mainly used for face detection.[6]

\subsection{Haar-like features}

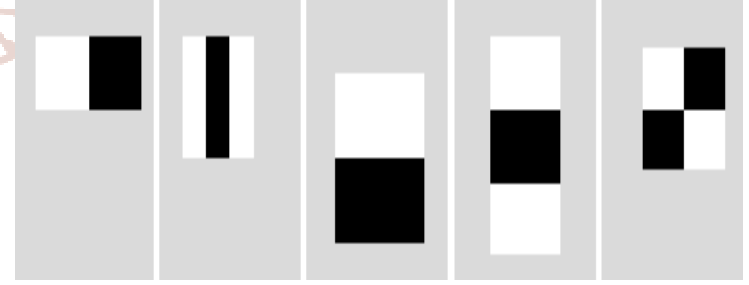

Figure 1.Five Haar like features

The size and position of the pattern's support can very provide its blank and white rectangles have the same dimension, border each other and keep their relative positions. Haar-like features are digital image features used in object recognition. They owe their name to 
their intuitive similarity with Haar wavelets and were used in the first real-time face detector. [5]

\section{FEATURES EXTRACTION}

Transformation of input data into a set of features. Features are distinctive properties of input patterns that help in different between the categories of an input pattern. Feature extraction involves reducing the amount of resources required to describe a large set of data. [1]

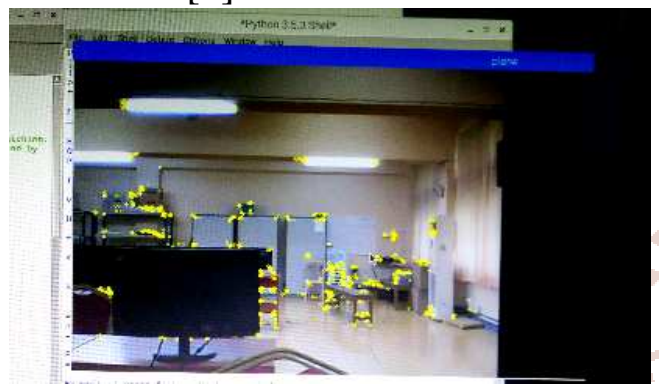

Figure2. Example of Feature Extraction

\section{FACE DETECTION}

The system is using Haar Classifier Method. We have developed three algorithms, for face detection from a given image, from a folder of images and for real time face detection. The following below figure 3 is for face detection system.

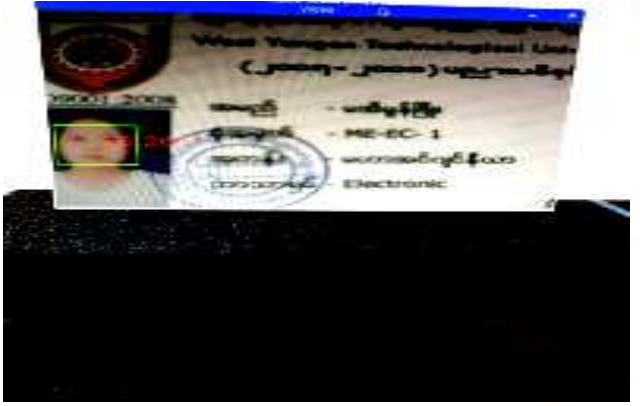

Figure3. (a) Face Detection by using Haar Classifier Method

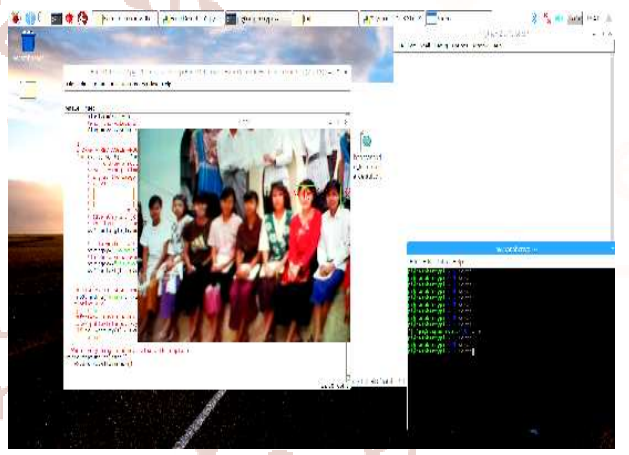

Figure3. (b) Face Detection by using Haar Classifier Method can be done at a level higher than that of the pixel. Identification of real objects, pseudo-objects, shadows, or actually finding anything of interest within the image, requires some form ; of segmentation.

Edge detection methods are used as a first step in the line detection process. Edge detection is also used to find complex object boundaries by marking the potential edge of points corresponding to places in an image where rapid changes in brightness occur. After these edge points have been marked, they can be merged to form lines and object outlines. Often people are confused about the difference between an edge and a line. [7]

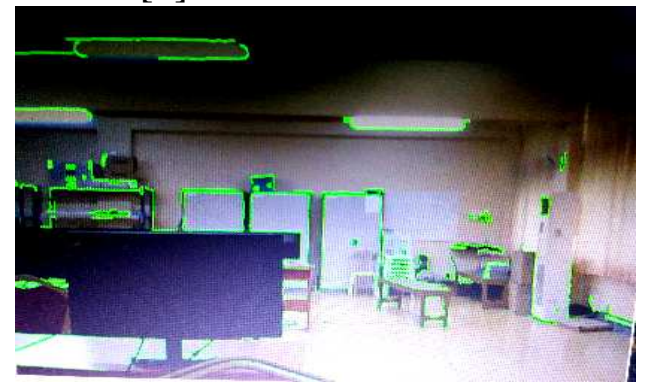

Figure2. Example of Segmentation and Edge/Line Detection

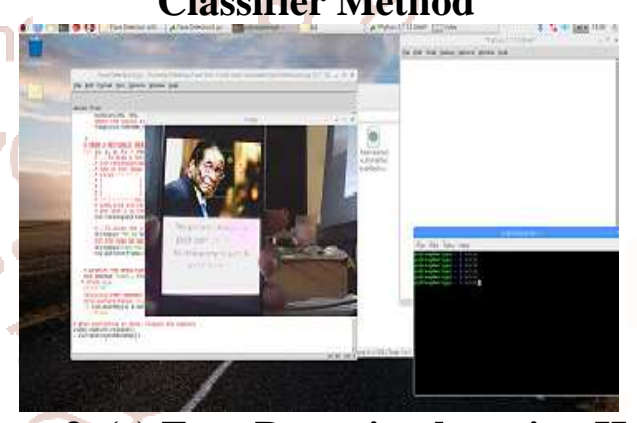

Figure3. (c) Face Detection by using Haar Classifier Method

\subsection{Aadvantages of by using Haar Classifier Method}

Haar-like classifier features are digital image features used in object recognition.

> A Haar-like feature considers adjacent rectangular regions at a specific location in a detection window, sums up the pixel intensities in each region and calculates the difference between these sums.

$>$ Advantage of a Haar-like feature over most other features is its calculation speed. Due to the use of integral images, a Haar-like feature of any size can be calculated in constant time (approximately 
International Journal of Trend in Scientific Research and Development (IJTSRD) ISSN: 2456-6470

60 microprocessor instructions for a 2-rectangle feature).

$>$ The main of cascading is achieving a good performance for both accuracy and timecomplexity, when you have a number of weak classifier.

$>$ The weak classifier must work at least with $51 \%$ accuracy. The cascade classifier rejects the many of samples in first node classifier with an efficient time.

$>$ High detection accuracy and low false positive rate.[4]

\section{EQUIPMENT OF EXPERIMENTAL TEST}
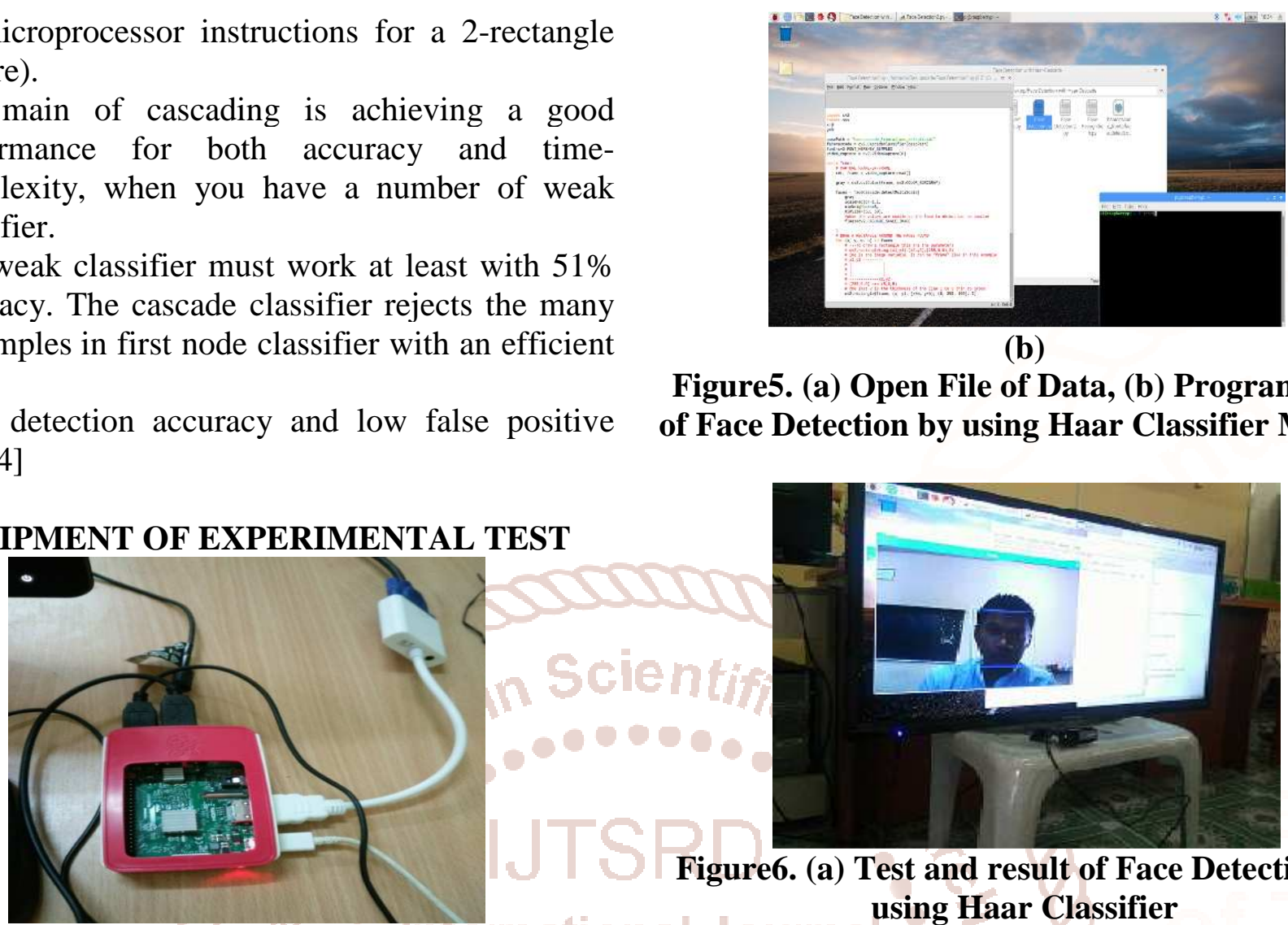

(b)

Figure5. (a) Open File of Data, (b) Programming of Face Detection by using Haar Classifier Method

Figure4. (a) Equipment of Test

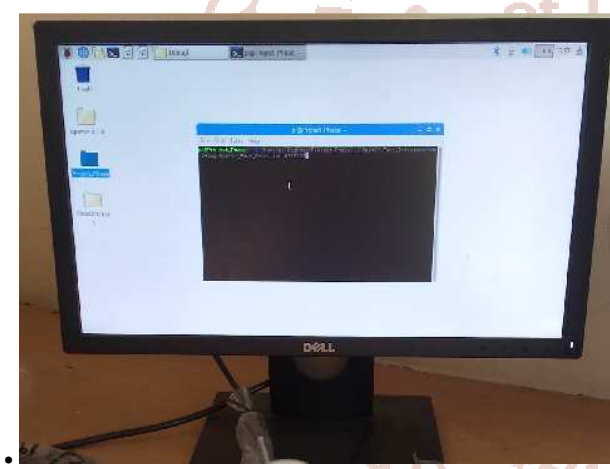

Figure4. (b) Equipment of Test

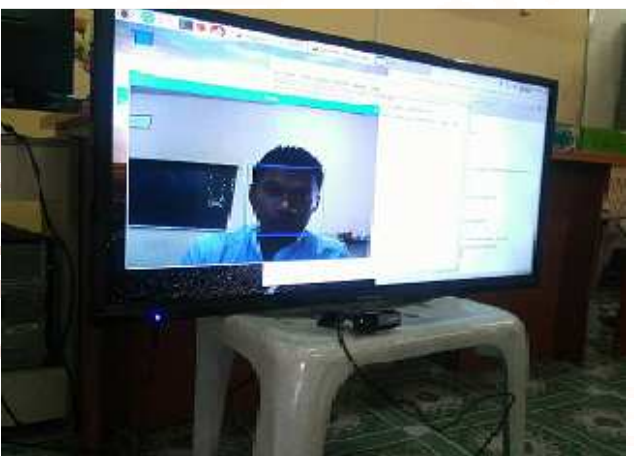

Figure6. (a) Test and result of Face Detection by using Haar Classifier

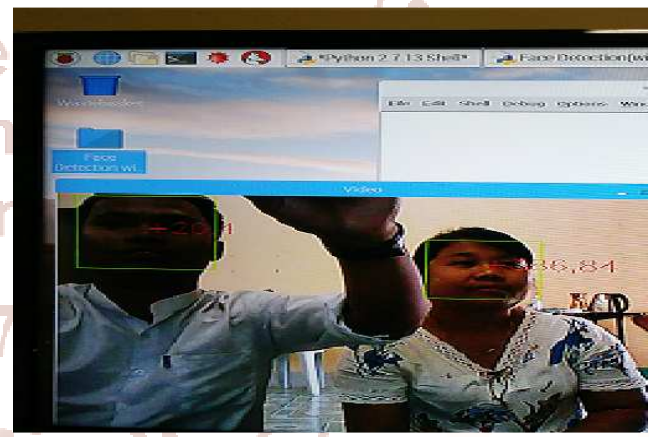

Figure6. (b) Test and result of Face Detection by using Haar Classifier

\section{CONCLUSION}

\section{TEST AND RESULTS}

In this section, tests and results of face detection and classification system using Haar Classifier Method are shown expressed. The performance of the algorithm as a whole is analyzed and discussed below the figure. The image pre-processing is shown in Figure 5.

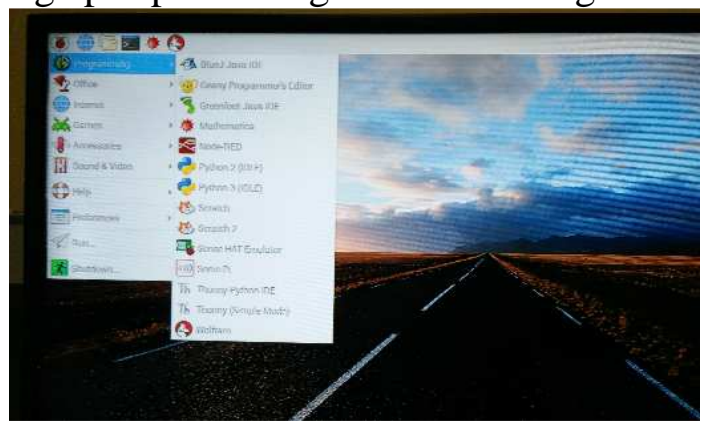

(a)
The smart security system has been aimed to design in such a way that it can fulfil the needs of the users for surveillance security area. Face detection security is very useful and essential important in many living together in the world. Using Haar Classifier Method, we have worse knowledge and sharing interface between human and computer software today.

\section{ACKNOWLEDGEMENTS}

Firstly, the author would like to acknowledge Dr. Kyi Soe, Rector of West Yangon Technological University, for his kind permission to carry out this research work. The authors would like to express their thanks to all persons who have given support during the preparation period of this paper. The authors 
would like to thank many colleagues from digital image processing research group of Department of Electronic Engineering of West Yangon Technological University. The author particularly wishes to acknowledge (Head of Electronic Department) Dr. Nay Win Zaw, my supervisor Daw Aye Aye Tun, my teacher U Myat Htut Zaw and all the teachers from Department of Electronic Engineering, West Yangon Technological University, for their support, encouragement and invaluable guidance in preparation of this research. The authors would like to express their thanks to all persons who have given support during the period of this research work.

\section{REFERENCE}

1. https://en.wikiwpedia.org/iki/feature_extractio

2. K.Raju1\& Dr. Y. Srinivasa Rao "Real time Implementation of Face Recognition System on
Raspberry Pi" International Journal of Engineering \& Technology, 7 (2.17) (2018) 85-89

3. https://en.wikipedia.org/wiki/Computer_vision

4. https://en.wikipedia.org/wiki/Haarlike_feature

5. Vandna Singh1, Dr. Vinod Shokeen2, Bhupendra Singh3 "Face Detection by Haar Cascade Classifier with Simple and Complex Backgrounds Images Using Open CV Implementation" International Journal of Advanced Technology in Engineering and Science, Volume No.01, Issue No. 12, December 2013

6. Nora Krasner, "Real-time face detection with Haar cascades"

7. SCOTT E UMBAUGH "Digital Image Processing and Analysis" "Human and Computer Vision with CVIP tools second edition"

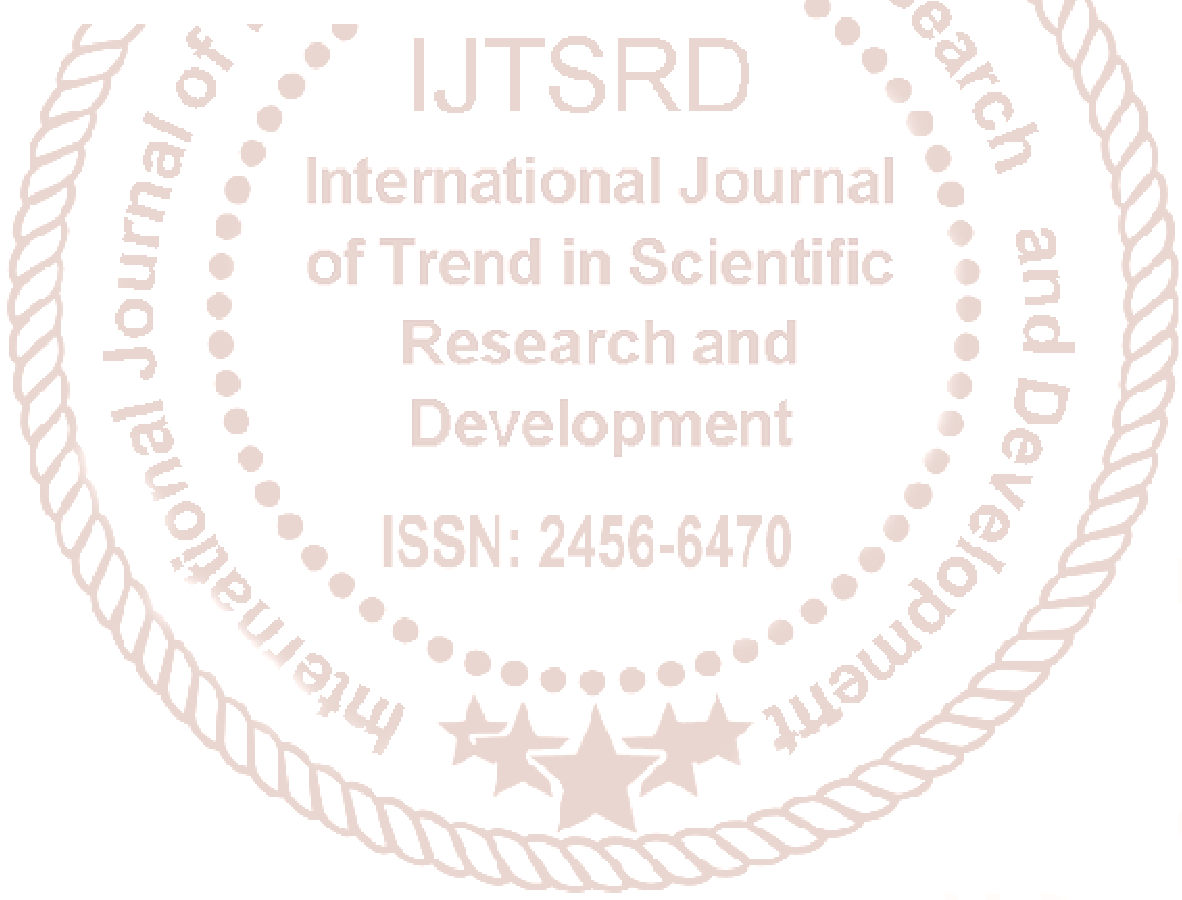

